\title{
CLASSIFICATION OF TERNARY EXTREMAL SELF-DUAL CODES OF LENGTH 28
}

\author{
MASAAKI HARADA, AKIHIRO MUNEMASA, AND BORIS VENKOV
}

\begin{abstract}
All 28-dimensional unimodular lattices with minimum norm 3 are known. Using this classification, we give a classification of ternary extremal self-dual codes of length 28 . Up to equivalence, there are 6,931 such codes.
\end{abstract}

\section{INTRODUCTION}

As described in 21, self-dual codes are an important class of linear codes for both theoretical and practical reasons. It is a fundamental problem to classify self-dual codes of modest length and determine the largest minimum weight among self-dual codes of that length. By the Gleason-Pierce theorem, there are nontrivial divisible self-dual codes over $\mathbb{F}_{q}$ for $q=2,3$ and 4 only, where $\mathbb{F}_{q}$ denotes the finite field of order $q$; this is one of the reasons why much work has been done concerning self-dual codes over these fields.

A ternary self-dual code $C$ of length $n$ is a code over $\mathbb{F}_{3}$ satisfying $C=C^{\perp}$ where the dual code $C^{\perp}$ of $C$ is defined as $C^{\perp}=\left\{x \in \mathbb{F}_{3}^{n} \mid x \cdot y=0\right.$ for all $\left.y \in C\right\}$ under the standard inner product $x \cdot y$. A ternary self-dual code of length $n$ exists if and only if $n \equiv 0(\bmod 4)$. It was shown in $[18$ that the minimum weight $d$ of a self-dual code of length $n$ is bounded by $d \leq 3[n / 12]+3$. If $d=3[n / 12]+3$, then the code is called extremal.

All ternary self-dual codes of length $\leq 20$ have been classified [5, 17, 20. At length 24 , the complete classification has not yet been done, but by showing that every ternary extremal self-dual code is generated by the rows of some Hadamard matrix of order 24 , it is shown that there are exactly two inequivalent extremal self-dual codes [16] (see [14 for known results on the classification).

The aim of this paper is to establish the following classification.

Theorem 1. There are exactly 6,931 inequivalent ternary extremal self-dual codes of length 28 .

Generator matrices of all codes can be obtained electronically from 11. All computer calculations in this paper were done using Magma [4].

Received by the editor January 29, 2008 and, in revised form, June 9, 2008.

2000 Mathematics Subject Classification. Primary 94B05; Secondary 11H71.

Key words and phrases. Extremal self-dual code, unimodular lattice, frame.

The work of the first and second authors was partially supported by the Sumitomo Foundation (Grant for Basic Science Research Projects, 050034). 


\section{SElF-DUAl CODES AND UNimodular LATtices}

Let $\mathbb{Z}_{k}$ be the ring of integers modulo $k$, where $k$ is a positive integer. Although we shall exclusively deal with the special case $k=3$ (and hence $\mathbb{Z}_{k}=\mathbb{F}_{3}$ ) in later sections, we discuss the general case here since the presentation remains the same for all $k$. A code $C$ of length $n$ over $\mathbb{Z}_{k}$ (or a $\mathbb{Z}_{k}$-code of length $n$ ) is a $\mathbb{Z}_{k}$-submodule of $\mathbb{Z}_{k}^{n}$. A code $C$ is self-dual if $C=C^{\perp}$ where the dual code $C^{\perp}$ of $C$ is defined as $C^{\perp}=\left\{x \in \mathbb{Z}_{k}^{n} \mid x \cdot y=0\right.$ for all $\left.y \in C\right\}$ under the standard inner product $x \cdot y$. Two $\mathbb{Z}_{k}$-codes $C$ and $C^{\prime}$ are equivalent if there exists a monomial $( \pm 1,0)$-matrix $P$ with $C^{\prime}=C \cdot P=\{x P \mid x \in C\}$. The automorphism group $\operatorname{Aut}(C)$ of $C$ is the group of all monomial $( \pm 1,0)$-matrices $P$ with $C=C \cdot P$.

A (Euclidean) lattice $L$ in dimension $n$ is integral if $L \subseteq L^{*}$, where the dual lattice $L^{*}$ is defined as $L^{*}=\left\{x \in \mathbb{R}^{n} \mid(x, y) \in \mathbb{Z}\right.$ for all $\left.y \in L\right\}$ under the standard inner product $(x, y)$. An integral lattice with $L=L^{*}$ is called unimodular. The norm of a vector $x$ is $(x, x)$. The minimum norm of $L$ is the smallest norm among all nonzero vectors of $L$. The theta series $\theta_{L}(q)$ of $L$ is the formal power series $\theta_{L}(q)=\sum_{x \in L} q^{(x, x)}=\sum_{m=0}^{\infty} N_{m} q^{m}$, where $N_{m}$ is the number of vectors of norm $m$. The kissing number is the second nonzero coefficient of the theta series, that is, the number of vectors of $L$ with minimum norm. Two lattices $L$ and $L^{\prime}$ are isomorphic, denoted $L \cong L^{\prime}$, if there exists an orthogonal matrix $A$ with $L^{\prime}=L \cdot A$. The automorphism group $\operatorname{Aut}(L)$ of $L$ is the group of all orthogonal matrices $A$ with $L=L \cdot A$.

For a $\mathbb{Z}_{k}$-code $C$ of length $n$,

$$
A_{k}(C)=\frac{1}{\sqrt{k}}\left\{\left(x_{1}, \ldots, x_{n}\right) \in \mathbb{Z}^{n} \mid\left(x_{1} \bmod k, \ldots, x_{n} \bmod k\right) \in C\right\}
$$

is a lattice, and $A_{k}(C)^{*}=A_{k}\left(C^{\perp}\right)$ holds (see e.g., [3, Lemma 3.1]). In particular, $C$ is self-dual if and only if $A_{k}(C)$ is unimodular. This construction of lattices from codes is called Construction A. Clearly, if $\mathbb{Z}_{k}$-codes $C$ and $C^{\prime}$ are equivalent, then the lattices $A_{k}(C)$ and $A_{k}\left(C^{\prime}\right)$ are isomorphic. Indeed, if $P$ is a monomial $( \pm 1,0)$-matrix and $C^{\prime}=C \cdot P$, then $A_{k}(C) \cdot P=A_{k}\left(C^{\prime}\right)$.

A set $\left\{f_{1}, \ldots, f_{n}\right\}$ of $n$ vectors $f_{1}, \ldots, f_{n}$ in an $n$-dimensional lattice $L$ with $\left(f_{i}, f_{j}\right)=k \delta_{i j}$ is called a $k$-frame of $L$, where $\delta_{i j}$ is the Kronecker delta. Clearly, $A_{k}(C)$ has a $k$-frame. Conversely, self-dual codes over $\mathbb{Z}_{k}$ correspond to $k$-frames in unimodular lattices. To state this precisely and more generally, we consider an arbitrary integral lattice $L$ in dimension $n$, and let $\mathcal{F}=\left\{f_{1}, \ldots, f_{n}\right\}$ be a $k$-frame of $L$. Consider the mapping

$$
\begin{aligned}
& \pi_{\mathcal{F}}: \frac{1}{k} \bigoplus_{i=1}^{n} \mathbb{Z} f_{i} \rightarrow \mathbb{Z}_{k}^{n} \\
& \pi_{\mathcal{F}}(x)=\left(\left(x, f_{i}\right) \bmod k\right)_{1 \leq i \leq n}
\end{aligned}
$$

Then $\operatorname{Ker} \pi_{\mathcal{F}}=\bigoplus_{i=1}^{n} \mathbb{Z} f_{i} \subset L$, so the code $C=\pi_{\mathcal{F}}(L)$ satisfies $\pi_{\mathcal{F}}^{-1}(C)=L$. This implies $A_{k}(C) \cong L$ and every code $C$ with $A_{k}(C) \cong L$ is obtained as $\pi_{\mathcal{F}}(L)$ for some $k$-frame $\mathcal{F}$ of $L$. In particular, if $L$ is unimodular, then $\pi_{\mathcal{F}}(L)$ is self-dual and every self-dual code $C$ with $A_{k}(C) \cong L$ is obtained in this way. 
Lemma 2. Let $L$ be an integral lattice in dimension $n$, and let $\mathcal{F}=\left\{f_{1}, \ldots, f_{n}\right\}$, $\mathcal{F}^{\prime}=\left\{f_{1}^{\prime}, \ldots, f_{n}^{\prime}\right\}$ be $k$-frames of $L$. Then the codes $\pi_{\mathcal{F}}(L)$ and $\pi_{\mathcal{F}^{\prime}}(L)$ are equivalent if and only if there exists an orthogonal matrix $P \in \operatorname{Aut}(L)$ such that

$$
\left\{ \pm f_{1}, \ldots, \pm f_{n}\right\} \cdot P=\left\{ \pm f_{1}^{\prime}, \ldots, \pm f_{n}^{\prime}\right\} .
$$

Proof. Let $C=\pi_{\mathcal{F}}(L), C^{\prime}=\pi_{\mathcal{F}^{\prime}}(L)$. Since $A_{k}(C \cdot Q)=A_{k}(C) \cdot Q$ for a monomial $( \pm 1,0)$-matrix $Q$, the codes $C$ and $C^{\prime}$ are equivalent if and only if $A_{k}(C) \cdot Q=$ $A_{k}\left(C^{\prime}\right)$ for some monomial $( \pm 1,0)$-matrix $Q$. Let $F$ and $F^{\prime}$ denote the $n \times n$ matrices whose row vectors consist of the elements of $\mathcal{F}$ and $\mathcal{F}^{\prime}$, respectively. Then

$$
A_{k}(C) \cdot \frac{1}{\sqrt{k}} F=A_{k}\left(C^{\prime}\right) \cdot \frac{1}{\sqrt{k}} F^{\prime}=L .
$$

This implies that the codes $C$ and $C^{\prime}$ are equivalent if and only if $\frac{1}{k} F^{T} Q F^{\prime} \in \operatorname{Aut}(L)$ for some monomial $( \pm 1,0)$-matrix $Q$. This occurs precisely when $F P=Q F^{\prime}$ for some $P \in \operatorname{Aut}(L)$.

\section{Methods of Classification}

In this section, we describe our approach for classifying ternary extremal self-dual codes of length 28 .

The minimum norm of a unimodular lattice in dimension 28 is at most 3 (see [6. Chapter 19]). We say that such a unimodular lattice with minimum norm 3 is optimal. There are 38 non-isomorphic optimal unimodular lattices in dimension 28 [2]. In [2], the 38 optimal unimodular lattices are denoted by $\mathbf{R}_{28,1}(\emptyset)$, $\mathbf{R}_{28,2}(\emptyset), \ldots, \mathbf{R}_{28,36}(\emptyset), \mathbf{R}_{28,37 e}(\emptyset), \mathbf{R}_{28,38 e}(\emptyset)$. These lattices have the following theta series:

$$
\begin{aligned}
& \theta_{\mathbf{R}_{28, i}(\emptyset)}(q)=1+2240 q^{3}+98280 q^{4}+\cdots(i=1,2, \ldots, 36), \\
& \theta_{\mathbf{R}_{28, i}(\emptyset)}(q)=1+1728 q^{3}+106472 q^{4}+\cdots(i=37 e, 38 e) .
\end{aligned}
$$

In order to distinguish lattices, some polynomial $m_{v}(x)$ is defined in 2, p. 239]. We remark that the polynomial $m_{v}(x)$ has a misprint, namely, " $\langle v, \alpha\rangle=1$ " should be " $\langle v, \alpha\rangle=-1 "$.

If $C$ is a self-dual code with minimum weight $d$, then $A_{3}(C)$ is a unimodular lattice with minimum norm $\min \{3, d / 3\}$. Thus, if $C$ is an extremal self-dual code of length 28 , then $A_{3}(C)$ is an optimal unimodular lattice. An extremal self-dual code $C$ of length 28 has the following weight enumerator:

$$
1+2184 y^{9}+78624 y^{12}+768096 y^{15}+2159976 y^{18}+\cdots .
$$

Since a codeword of weight 9 gives a vector of norm 3 in the lattice $A_{3}(C), A_{3}(C)$ has exactly $2184+2 \cdot 28$ vectors of norm 3 . Hence we have the following:

Lemma 3. Let $C$ be a ternary extremal self-dual code of length 28. Then the lattice $A_{3}(C)$ has theta series (1).

Lemma 4. Let $L$ be an optimal unimodular lattice in dimension 28 having a 3frame $\left\{u_{1}, \ldots, u_{28}\right\}$. Let $L_{0}$ be the even sublattice of the lattice $L$. If $u \in L_{0}^{*} \backslash L$, then

$$
u=\frac{1}{6} \sum_{i=1}^{28} \lambda_{i} u_{i}
$$

for some odd integers $\lambda_{1}, \ldots, \lambda_{28}$. 
Proof. Follows from [10, Lemma 2].

Lemma 5. Let $C$ be a ternary extremal self-dual code of length 28. Let $\left\{u_{1}, \ldots, u_{28}\right\}$ be a 3-frame of $L=A_{3}(C)$ coming from $C$. Let $L_{0}$ be the even sublattice of the lattice $L$. Then $L_{0}^{*}$ has minimum norm 3. Moreover, for any vector $u \in L_{0}^{*} \backslash L$ of norm 3 , there exists a unique $i_{0} \in\{1, \ldots, 28\}$ such that

$$
\left|\left(u, u_{i}\right)\right|= \begin{cases}\frac{3}{2} & \text { if } i=i_{0}, \\ \frac{1}{2} & \text { otherwise. }\end{cases}
$$

Proof. Since $C$ has minimum weight $9, L$ has minimum norm 3 . Hence $L_{0}$ has minimum norm 4 (see also [10, Lemma 1]). Let $u \in L_{0}^{*} \backslash L$ and write $u$ as in (3). Then by Lemma 4, we have

$$
\begin{aligned}
28 & \leq \sum_{i=1}^{28} \lambda_{i}^{2} \\
& =\frac{1}{3} \sum_{i=1}^{28}\left(\lambda_{i} u_{i}, \lambda_{i} u_{i}\right) \\
& =\frac{1}{3}(6 u, 6 u) \\
& =12(u, u) .
\end{aligned}
$$

Since $(u, u) \in \mathbb{Z}$ we have $(u, u) \geq 3$, and equality holds if and only if there exists a unique $i_{0} \in\{1, \ldots, 28\}$ such that

$$
\left|\lambda_{i}\right|= \begin{cases}3 & \text { if } i=i_{0} \\ 1 & \text { otherwise }\end{cases}
$$

and the assertion follows.

Remark 6. By Lemma 3, $L$ has theta series (11). From [6, p. xlv], the shadow $L_{0}^{*} \backslash L$ has minimum norm 3 . This gives an alternative proof of the assertion that $L_{0}^{*}$ has minimum norm 3 .

For a lattice $L$, a vector $u$ not necessarily in $L$, an integer $m$ and a real number $c$, set

$$
L_{m, c}(u)=\{v \mid v \in L,(v, v)=m,(u, v)= \pm c\} .
$$

Lemma 7. Let $L$ be an optimal unimodular lattice in dimension 28 . Let $S_{3}$ be the set of vectors of norm 3 in $L_{0}^{*} \backslash L$. Let $u_{1}, u_{2}$ be orthogonal vectors of norm 3 in $L$. If there exists a 3-frame containing $u_{1}$ and $u_{2}$, then

$$
\left\{u_{1}, u_{2}\right\} \not \subset L_{3,3 / 2}(u) \quad \text { for all } u \in S_{3} .
$$

Proof. Immediate from Lemma 5 .

Let $L$ be a 28-dimensional optimal unimodular lattice with kissing number 2240 . Let $L_{3}$ be the set $\{\{x,-x\} \mid(x, x)=3, x \in L\}$ and let $S_{3}$ be the set $\{x \mid(x, x)=3, x \in$ $\left.L_{0}^{*} \backslash L\right\}$. We define the simple undirected graph $\Gamma$, whose set of vertices is the set of 1120 pairs in $L_{3}$ and two vertices $\{x,-x\},\{y,-y\} \in L_{3}$ are adjacent if $(x, y)=0$ and $\{x, y\} \not \subset L_{3,3 / 2}(u)$ for any $u \in S_{3}$. It follows that the 3 -frames are precisely the 28 -cliques in the graph $\Gamma$. We could have defined $\Gamma$ as the "orthogonality graph," by simply joining two vertices $\{x,-x\},\{y,-y\} \in L_{3}$ whenever $(x, y)=0$. The effect 
of Lemma 7 is to remove those edges from the "orthogonality graph" which cannot be contained in 3-frames. This speeds up the computations considerably. It is clear that $\operatorname{Aut}(L)$ acts on the graph $\Gamma$ as automorphisms, and Lemma 2 implies that the $\operatorname{Aut}(L)$-orbits on the set of 28 -cliques in $\Gamma$ are in one-to-one correspondence with the equivalence classes of codes $C$ satisfying $A_{3}(C) \cong L$. Therefore, the classification of such codes reduces to finding a set of representatives of 28 -cliques in $\Gamma$ up to the action of $\operatorname{Aut}(L)$. This computation was performed using MAGMA, the results were then converted to 3-frames, and then to ternary extremal self-dual codes of length 28. In this way, by considering all 28-dimensional optimal unimodular lattices with kissing number 2240, we have all inequivalent extremal self-dual codes of length 28 by Lemma 3 ,

Remark 8. From the odd Leech lattice which is the unique odd unimodular lattice with minimum norm 3 in dimension 24, we can define the "orthogonality graph" as above. Classifying 24-cliques up to the automorphism group of the odd Leech lattice, we have verified that there are exactly two 3 -frames in the odd Leech lattice up to conjugacy under the automorphism group. This confirms the known classification (see [16]) of ternary extremal self-dual codes of length 24.

\section{Results of ClassificAtion}

By the approach described in Section 3. we completed the classification of ternary extremal self-dual codes of length 28 . The number $F_{i}(i=1,2, \ldots, 36)$ of 3 -frames in $\mathbf{R}_{28, i}(\emptyset)$ is listed in Table 1 . The number $N_{i}(i=1,2, \ldots, 36)$ of inequivalent extremal self-dual codes $C$ with $A_{3}(C) \cong \mathbf{R}_{28, i}(\emptyset)$ is also listed in Table 1. The last column (\# Aut, $N_{i}$ (\# Aut)) in Table 1 lists the number $N_{i}$ (\# Aut) of the codes whose automorphism groups have order \# Aut. Therefore, we have Theorem 1. As a corollary, we have the following:

Corollary 9. A 28-dimensional optimal unimodular lattice $L$ can be constructed from some ternary extremal self-dual code of length 28 by Construction $A$ if and only if $L$ is isomorphic to $\mathbf{R}_{28, i}(\emptyset)$ for some $i=1,2, \ldots, 27,29, \ldots, 33,35,36$.

We investigate the previously known extremal self-dual codes of length 28 . Huffman [13] showed that there are 14 and 5 inequivalent extremal self-dual $[28,14,9]$ codes with automorphisms of orders 7 and 13, respectively. Denote the 14 codes with automorphisms of order 7 by $H_{7,1}, \ldots, H_{7,14}$, and the 5 codes with automorphisms of order 13 by $H_{13,1}, \ldots, H_{13,5}$, according to the order in [13, Theorem 4]. However, he did not check if there is a pair of equivalent codes $H_{7, i}$ and $H_{13, j}$ among these codes. We have verified that the codes $H_{7,2}$ and $H_{13,2}$ are equivalent and the codes $H_{7,14}$ and $H_{13,3}$ are equivalent.

In [8, 16 inequivalent extremal self-dual codes which are inequivalent to any of the codes in [13] are constructed and these codes are denoted by $C_{28,1}, \ldots, C_{28,16}$ in 8]. We remark that the existence of some extremal ternary self-dual code of length 28 with trivial automorphism group is announced in [9] and one example of such a code is given. However, we have verified that the code with generator matrix $G_{28}$ given in [9] is equivalent to $C_{28,1}$. Hence 33 inequivalent extremal self-dual codes are previously known explicitly. We have verified that all of these 33 codes appear in the present classification, and we list the order \# Aut $(C)$ of the automorphism group and the lattice $\mathbf{R}_{28, i}(\emptyset)$ with $A_{3}(C) \cong \mathbf{R}_{28, i}(\emptyset)$ in Table 2 
TABLE 1. Ternary extremal self-dual codes of length 28

\begin{tabular}{c|r|r|l}
\hline$i$ & \multicolumn{1}{|c|}{$F_{i}$} & \multicolumn{1}{|c}{$N_{i}$} & \multicolumn{1}{|c}{$\left(\#\right.$ Aut,$N_{i}$ (\# Aut) $)$} \\
\hline 1 & 4144 & 1036 & $(2,1036)$ \\
2 & 4804 & 735 & $(2,501),(4,166),(8,64),(16,4)$ \\
3 & 4218 & 589 & $(2,474),(4,98),(8,17)$ \\
4 & 4088 & 575 & $(2,448),(4,125),(8,2)$ \\
5 & 4728 & 667 & $(2,516),(4,149),(8,2)$ \\
6 & 3896 & 558 & $(2,417),(4,139),(8,2)$ \\
7 & 4420 & 376 & $(2,364),(4,3),(6,8),(12,1)$ \\
8 & 4296 & 333 & $(2,213),(4,102),(8,18)$ \\
9 & 4768 & 298 & $(2,298)$ \\
10 & 3328 & 208 & $(2,208)$ \\
11 & 4480 & 328 & $(2,237),(4,81),(8,10)$ \\
12 & 4184 & 166 & $(2,102),(4,51),(8,13)$ \\
13 & 5268 & 154 & $(2,83),(4,40),(6,6),(8,13),(12,6),(16,1),(24,4),(48,1)$ \\
14 & 5656 & 180 & $(2,64),(4,102),(6,2),(8,4),(12,6),(24,2)$ \\
15 & 5744 & 176 & $(2,56),(4,74),(8,35),(16,5),(56,5),(112,1)$ \\
16 & 4720 & 76 & $(2,44),(4,28),(8,4)$ \\
17 & 5080 & 84 & $(2,30),(4,40),(6,3),(8,2),(12,8),(24,1)$ \\
18 & 5968 & 83 & $(2,49),(4,15),(6,13),(8,5),(24,1)$ \\
19 & 3360 & 37 & $(2,19),(4,11),(8,7)$ \\
20 & 4608 & 57 & $(2,18),(4,33),(8,6)$ \\
21 & 5360 & 44 & $(2,25),(4,15),(8,4)$ \\
22 & 3100 & 16 & $(2,15),(4,1)$ \\
23 & 6336 & 36 & $(2,16),(4,15),(8,5)$ \\
24 & 5568 & 22 & $(2,9),(4,9),(8,4)$ \\
25 & 9432 & 36 & $(2,3),(4,7),(6,1),(8,11),(12,2),(16,3),(24,6),(48,3)$ \\
26 & 18688 & 26 & $(4,4),(6,5),(8,3),(12,6),(16,1),(24,6),(48,1)$ \\
27 & 5120 & 4 & $(6,4)$ \\
28 & 0 & 0 & \\
29 & 5472 & 3 & $(6,2),(54,1)$ \\
30 & 41856 & 9 & $(4,1),(6,1),(12,1),(16,1),(18,1),(24,3),(72,1)$ \\
31 & 14400 & 6 & $(24,2),(48,3),(336,1)$ \\
32 & 16960 & 5 & $(28,3),(52,2)$ \\
33 & 36864 & 4 & $(24,2),(48,1),(336,1)$ \\
34 & 0 & 0 & \\
35 & 230400 & 1 & $(3024,1)$ \\
36 & 12908160 & 3 & $(2184,1),(4212,1),(117936,1)$ \\
\hline & & & \\
\hline & &
\end{tabular}

For those lattices $\mathbf{R}_{28, i}(\emptyset)$ which are not listed in Table 2 we list a code $C_{i}$ with $\mathbf{R}_{28, i}(\emptyset) \cong A_{3}\left(C_{i}\right)$ where the rows of the matrix $M_{i}$ in a generator matrix $\left(I, M_{i}\right)$ of $C_{i}$ are given in Table 3 . The order \# Aut $\left(C_{i}\right)$ of the automorphism group of $C_{i}$ is also listed.

The lattice $\mathbf{R}_{28,35}(\emptyset)$ was constructed by Nebe [19], and it can be given simply as

$$
\Lambda=E_{8} \wedge E_{8}
$$

where $E_{8}$ denotes the root lattice of type $E_{8}$. The unique code $C$ with $A_{3}(C) \cong \Lambda$ can be constructed from a 3 -frame of the lattice $\Lambda$, which we shall now describe.

Let $e_{1}, \ldots, e_{9}$ denote an orthonormal basis of the vector space $\mathbb{R}^{9}$. The $\mathbb{Z}$-linear span of $\left\{e_{i}-e_{j} \mid 1 \leq i<j \leq 9\right\}$ is known as the $A_{8}$-lattice. For a subset $T$ of $\{1, \ldots, 9\}$, we denote by $e_{T}$ the vector $\sum_{i \in T} e_{i}$, and by $\bar{T}$ the complementary set of $T$ in $\{1, \ldots, 9\}$. Let $\mathcal{T}$ denote the set of all 3 -subsets of the set $\{1, \ldots, 9\}$. Then, together with the 72 roots of $A_{8}$, the $2\left(\begin{array}{l}9 \\ 3\end{array}\right)=168 \operatorname{roots}\left\{ \pm \frac{1}{3}\left(2 e_{T}-e_{\bar{T}}\right) \mid T \in\right.$ $\mathcal{T}\}$ form the root system $E_{8}$. The group $\operatorname{PSL}(2,8)$ acts triply transitively on the 
TABLE 2. The automorphism groups and lattices of known codes

\begin{tabular}{c|r|c||c|r|c}
\hline Codes $C$ & \# Aut $(C)$ & $\mathbf{R}_{28, i}(\emptyset)$ & Codes $C$ & \# Aut $(C)$ & $\mathbf{R}_{28, i}(\emptyset)$ \\
\hline$H_{7,1}$ & 56 & $\mathbf{R}_{28,15}(\emptyset)$ & $C_{28,1}$ & 2 & $\mathbf{R}_{28,16}(\emptyset)$ \\
$H_{7,2}$ & 2184 & $\mathbf{R}_{28,36}(\emptyset)$ & $C_{28,2}$ & 4 & $\mathbf{R}_{28,12}(\emptyset)$ \\
$H_{7,3}$ & 28 & $\mathbf{R}_{28,32}(\emptyset)$ & $C_{28,3}$ & 2 & $\mathbf{R}_{28,11}(\emptyset)$ \\
$H_{7,4}$ & 56 & $\mathbf{R}_{28,15}(\emptyset)$ & $C_{28,4}$ & 24 & $\mathbf{R}_{28,17}(\emptyset)$ \\
$H_{7,5}$ & 28 & $\mathbf{R}_{28,32}(\emptyset)$ & $C_{28,5}$ & 2 & $\mathbf{R}_{28,9}(\emptyset)$ \\
$H_{7,6}$ & 28 & $\mathbf{R}_{28,32}(\emptyset)$ & $C_{28,6}$ & 4 & $\mathbf{R}_{28,5}(\emptyset)$ \\
$H_{7,7}$ & 56 & $\mathbf{R}_{28,15}(\emptyset)$ & $C_{28,7}$ & 2 & $\mathbf{R}_{28,2}(\emptyset)$ \\
$H_{7,8}$ & 112 & $\mathbf{R}_{28,15}(\emptyset)$ & $C_{28,8}$ & 4 & $\mathbf{R}_{28,6}(\emptyset)$ \\
$H_{7,9}$ & 3024 & $\mathbf{R}_{28,35}(\emptyset)$ & $C_{28,9}$ & 2 & $\mathbf{R}_{28,2}(\emptyset)$ \\
$H_{7,10}$ & 56 & $\mathbf{R}_{28,15}(\emptyset)$ & $C_{28,10}$ & 2 & $\mathbf{R}_{28,6}(\emptyset)$ \\
$H_{7,11}$ & 56 & $\mathbf{R}_{28,15}(\emptyset)$ & $C_{28,11}$ & 4 & $\mathbf{R}_{28,5}(\emptyset)$ \\
$H_{7,12}$ & 336 & $\mathbf{R}_{28,33}(\emptyset)$ & $C_{28,12}$ & 8 & $\mathbf{R}_{28,2}(\emptyset)$ \\
$H_{7,13}$ & 336 & $\mathbf{R}_{28,31}(\emptyset)$ & $C_{28,13}$ & 8 & $\mathbf{R}_{28,13}(\emptyset)$ \\
$H_{7,14}$ & 117936 & $\mathbf{R}_{28,36}(\emptyset)$ & $C_{28,14}$ & 2 & $\mathbf{R}_{28,3}(\emptyset)$ \\
$H_{13,1}$ & 4212 & $\mathbf{R}_{28,36}(\emptyset)$ & $C_{28,15}$ & 2 & $\mathbf{R}_{28,2}(\emptyset)$ \\
$H_{13,4}$ & 52 & $\mathbf{R}_{28,32}(\emptyset)$ & $C_{28,16}$ & 4 & $\mathbf{R}_{28,3}(\emptyset)$ \\
$H_{13,5}$ & 52 & $\mathbf{R}_{28,32}(\emptyset)$ & & & \\
\hline
\end{tabular}

projective line $\mathbb{F}_{8} \cup\{\infty\}$. Identifying $\mathbb{F}_{8} \cup\{\infty\}$ with $\{1, \ldots, 9\}$, we regard $\operatorname{PSL}(2,8)$ as a permutation group on the set $\{1, \ldots, 9\}$. The group $\operatorname{PSL}(2,8)$ has a unique conjugacy class $\mathcal{C}$ of fixed-point-free elements of order 3. This conjugacy class $\mathcal{C}$ contains 28 pairs of elements and their inverses. Let $x \in \mathcal{C}$, and let $T_{1}, T_{2}, T_{3}$ be the $\langle x\rangle$-orbits on $\{1, \ldots, 9\}$. Define $f_{x} \in \Lambda$ by

$$
f_{x}=\frac{1}{3}\left(e_{T_{1}} \wedge e_{T_{2}}+e_{T_{2}} \wedge e_{T_{3}}+e_{T_{3}} \wedge e_{T_{1}}\right) .
$$

Note that $f_{x}$ is not uniquely determined by $x$, but determined up to sign. The set $\left\{ \pm f_{x} \mid x \in \mathcal{C}\right\}$ forms a 3 -frame in the lattice $\Lambda$. One obtains an extremal ternary self-dual code of length 28 from this 3 -frame of $\Lambda$.

Finally, we remark that the lattice $\mathbf{R}_{28,36}(\emptyset)$ was first constructed in [1, and 3 -frames of the lattice $\mathbf{R}_{28,36}(\emptyset)$ correspond to symplectic spreads in $\mathbb{F}_{3}^{6}$, as pointed out in [2, Remark 2.1]. Consequently, our classification gives an alternative proof of the classification of symplectic spreads in $\mathbb{F}_{3}^{6}$, given in 7 .

TABLE 3. Some generator matrices of extremal self-dual codes

\begin{tabular}{c|l|c}
\hline$i$ & \multicolumn{1}{|c|}{$M_{i}$} & \# Aut $\left(C_{i}\right)$ \\
\hline 1 & $12211011201012,10210021121122,11102212101220,10002220012101$, & 2 \\
& $00212001122200,02222011011000,21022211212001,22222221220200$, & \\
& $02010102210102,22202122120011,01212120122022,12221120120120$, & \\
& 02121021200020,01210002020222 & \\
\hline 4 & $22222002021111,01012111210222,12101200211221,20221222012011$, & 8 \\
& $12000020211012,20201121222210,20202101200022,11222221222111$, & \\
& $01211000210201,00102111212000,02122011001001,20110001222020$, & \\
& 02000102201222,00222022122212 & 12 \\
\hline 7 & $00001210210122,22121101220202,00220222212222,02012002211001$, & \\
& $01122210010100,10102000022221,01212012020002,22222210102101$, & \\
& $00101101110012,20120000101121,11010012200201,21222122112211$, & \\
& 20212202211210,22211010112210 & \\
\hline 8 & $00122221211210,22210220110221,20102111111202,01122010200220$, & \\
& $00022121112122,20210121121022,22012200212122,11121020222021$, & \\
& $20222121201101,02211111220012,02212112001111,21200110112122$, & \\
& 00011002012222,11202200012100 & \\
\hline
\end{tabular}


TABLE 3. Some generator matrices of extremal self-dual codes (continued)

\begin{tabular}{|c|c|c|}
\hline$i$ & $M_{i}$ & $\# \operatorname{Aut}\left(C_{i}\right)$ \\
\hline 10 & $\begin{array}{l}\text { 02010220110021, 21201022112201, 00110120101101, 21210001211211, } \\
21122022100222,20221100212221,01022111112110,01220112101122, \\
00122212012000,21120011202112,02112002002220,22201111211001, \\
12012222012201,22200021200202\end{array}$ & 2 \\
\hline 14 & $\begin{array}{l}\text { 02220020101110, 00121002102210, 22210202112022, } 12221100121101 \text {, } \\
21011111210102,00211021100011,22110021121012,21102211022220 \text {, } \\
01002002111110,21011002201200,00212211022111,11122201110210 \text {, } \\
11101210102212,01121212002121\end{array}$ & 24 \\
\hline 18 & $\begin{array}{l}21211122212212,02221000210120,22102201111210,02111210220221, \\
21211220101102,11012122111002,20220100110021,10020001210222, \\
00221122012121,02110000202111,21222222111221,11001112021212, \\
02212101110000,02201220000111\end{array}$ & 24 \\
\hline 19 & $\begin{array}{l}\text { 10102002022202, 21220200100021, 00002011012211, 02102220111000, } \\
02012212202221,11100210210001,00111201002201,21120210012122 \text {, } \\
20010022011011,20120112211101,20200201120110,22222100111201 \text {, } \\
02201201012010,01121110111021\end{array}$ & 8 \\
\hline 20 & $\begin{array}{l}20222002202200,02000220120212,02212222102202,20001112200201, \\
00202202101120,10001212111000,11010221222202,20120221212220, \\
12201002111111,21202022221110,22211120002122,20022022020101, \\
12212020112021,11021011001020\end{array}$ & 8 \\
\hline 21 & $\begin{array}{l}11011212011220,10002202120102,12010100022102,10110221221011, \\
10112021120222,21110210110222,12111020020001,21211121001120, \\
10212222102012,01100000111121,00220220221200,00211210010201, \\
21100122112201,11222121000212\end{array}$ & 8 \\
\hline 22 & $\begin{array}{l}\text { 02211201222021, 10011021200102, 12221111112121, 00121200211001, } \\
21201111210102,01020021201120,00221222011212,20021221000220 \text {, } \\
\text { 20202102020101, 12220100000111, 02111100020201, 11122021202012, } \\
12210211022110,00102101101201\end{array}$ & 4 \\
\hline 23 & $\begin{array}{l}01110221001020,11101211001111,10010110011202,01121221012102, \\
22111010112021,21012220020010,12102000220102,20110222102221, \\
01211122210101,22210012222220,02202101011020,02000102121011, \\
00202210111200,21100220112000\end{array}$ & 8 \\
\hline 24 & $\begin{array}{l}00111220000122,00220011012110,10100022222002,00121212012221, \\
22122111001110,12112001100010,11200122200100,00121022210100, \\
11201012001002,12112122210001,01212010222111,12202101112021, \\
20202200211020,01202221000202\end{array}$ & 8 \\
\hline 25 & $\begin{array}{l}21222200220000,12011220000021,20020122101010,20102102121211, \\
00012002212022,01120200110220,20221112010000,10200221101100, \\
22121000112211,02012110100101,22021112201102,21120210121011, \\
20110021200110,11000102022201\end{array}$ & 48 \\
\hline 26 & $\begin{array}{l}20002202001122,11100202121112,00221102212212,21010012012010, \\
10010101210202,00110011002111,12021011201111,20011020222020, \\
21121120102120,11001120212222,00111212100001,22220100122112, \\
22211010221102,21220000100221\end{array}$ & 48 \\
\hline 27 & $\begin{array}{l}01121210201122,12212010222110,11201111001121,12121111020201, \\
01200202121010,20012201221221,10201222212202,20111100021200, \\
22101110110000,21222020122101,10221012211120,10100010201111, \\
11020021210020,00001021122102\end{array}$ & 6 \\
\hline 29 & $\begin{array}{l}12021100210100,10102100012102,21022101101222,22220111100211, \\
21001220122111,02202022101100,22011011201212,20011022222122, \\
22110110012212,10122001002220,11001020010122,12102021221210, \\
02201202002220,02120100022120\end{array}$ & 54 \\
\hline 30 & $\begin{array}{l}00202020211102,20001201001121,01120020210110,22020102002120, \\
21002011010021,20011012021001,22200210222211,02200022021201, \\
10102121221012,12121120122002,20022011120200,01010220202011, \\
00110121022001,01112200212122\end{array}$ & 72 \\
\hline
\end{tabular}




\section{Covering RAdi And 3-Designs}

The covering radius $R(C)$ of a code $C$ of length $n$ is the smallest integer $R$ such that spheres of radius $R$ around codewords of $C$ cover the space $\mathbb{F}_{3}^{n}$. For linear codes, the covering radius is the largest weight of all coset leaders of the code. The covering radius is a basic and important geometric parameter of a code. Let $C$ be an extremal self-dual code of length 28 . Then, by the sphere-covering bound and the Delsarte bound, it is known that $R(C)=6$ or 7 (see [12, Table 1]). Using our classification, explicit calculations show the following:

Proposition 10. Every extremal self-dual code of length 28 has covering radius 7.

A $t$ - $(v, k, \lambda)$ design is a set $X$ of $v$ points together with a collection $\mathcal{B}$ of $k$ subsets of $X$ called blocks such that every $t$-subset of $X$ is contained in exactly $\lambda$ blocks. Two designs with the same parameters are isomorphic if there is a bijection between their point sets that maps the blocks of the first design into blocks of the second design. The automorphism group $\operatorname{Aut}(D)$ of a $t$-design $D$ is the group of all isomorphisms of $D$ with itself. If a $3-(28,9, \lambda)$ design exists, then $\lambda$ must be divisible by 28 (see [15, p. 57]). Hence $3-(28,9,28)$ designs have the smallest $\lambda$ among $3-(28,9, \lambda)$ designs.

By the Assmus-Mattson theorem, the supports of the codewords of minimum weight in an extremal self-dual code of length 28 form a $3-(28,9,28)$ design. We have verified that the $6,9313-(28,9,28)$ designs obtained from the extremal self-dual codes are non-isomorphic. Thus we have the following:

Proposition 11. There are at least 6,931 non-isomorphic 3-(28,9, 28) designs.

Remark 12. We have verified that every extremal self-dual code of length 28 is generated by the codewords of minimum weight.

For every extremal self-dual code $C$ of length 28 , we have verified that the order of the automorphism group of the $3-(28,9,28)$ design obtained from $C$ is half of the order of the automorphism group of $C$.

\section{ACKNOWLEDGMENT}

The authors would like to thank Gabriele Nebe for helpful discussions.

\section{REFERENCES}

[1] R. Bacher and B. Venkov, Lattices and association schemes: a unimodular example without roots in dimension 28, Ann. Inst. Fourier (Grenoble) 45 (1995), 1163-1176. MR1370742 (96j:11093)

[2] R. Bacher and B. Venkov, Réseaux entiers unimodulaires sans racines en dimensions 27 et 28, Réseaux euclidiens, designs sphériques et formes modulaires, 212-267, Monogr. Enseign. Math., 37, Enseignement Math., Geneva, 2001. MR1878751(2003a:11082)

[3] C. Bachoc, T.A. Gulliver and M. Harada, Isodual codes over $\mathbb{Z}_{2 k}$ and isodual lattices, J. Algebraic Combin. 12 (2000), 223-240. MR1803233 (2001j:94052)

[4] W. Bosma and J. Cannon, Handbook of Magma Functions, Department of Mathematics, University of Sydney, Available online at http://magma.maths.usyd.edu.au/magma/.

[5] J.H. Conway, V. Pless and N.J.A. Sloane, Self-dual codes over GF(3) and GF(4) of length not exceeding 16, IEEE Trans. Inform. Theory 25 (1979), 312-322. MR528009(80h:94026)

[6] J.H. Conway and N.J.A. Sloane, Sphere Packing, Lattices and Groups (3rd ed.), SpringerVerlag, New York, 1999. MR1662447(2000b:11077)

[7] U. Dempwolff, Translation planes of order 27, Des. Codes Cryptogr. 4 (1994), 105-121. MR.1268564 (95a:51012) 
[8] M. Harada, New extremal ternary self-dual codes, Australas. J. Combin. 17 (1998), 133-145. MR:1626295 (99c:94043)

[9] M. Harada, An extremal ternary self-dual $[28,14,9]$ code with a trivial automorphism group, Discrete Math. 239 (2001), 121-125. MR.1850990 (2002m:94058)

[10] M. Harada, M. Kitazume and M. Ozeki, Ternary code construction of unimodular lattices and self-dual codes over $\mathbb{Z}_{6}$, J. Algebraic Combin. 16 (2002), 209-223. MR1943589(2004b:11099)

[11] M. Harada and A. Munemasa, Database of Self-Dual Codes, Available online at http://www.math.is.tohoku.ac.jp/ ${ }^{\sim}$ munemasa/selfdualcodes.htm.

[12] M. Harada, M. Ozeki and K. Tanabe, On the covering radius of ternary extremal self-dual codes, Des. Codes Cryptogr. 33 (2004), 149-158. MR2080361(2005d:94214)

[13] W.C. Huffman, On extremal self-dual ternary codes of lengths 28 to 40, IEEE Trans. Inform. Theory 38 (1992), 1395-1400. MR1168760 (93b:94030)

[14] W.C. Huffman, On the classification and enumeration of self-dual codes, Finite Fields Appl. 11 (2005), 451-490. MR2158773 (2006h:94253)

[15] D.L. Kreher, " $t$-Designs, $t \geq 3$," The CRC Handbook of Combinatorial Designs, C.J. Colbourn and J.H. Dinitz (Editors), CRC Press, Boca Raton, 1996, pp. 47-66. MR1392993 (97a:05001)

[16] J.S. Leon, V. Pless and N.J.A. Sloane, On ternary self-dual codes of length 24, IEEE Trans. Inform. Theory 27 (1981), 176-180. MR633414 (83c:94020)

[17] C.L. Mallows, V. Pless and N.J.A. Sloane, Self-dual codes over GF(3), SIAM J. Appl. Math. 31 (1976), 649-666. MR0441541 (55:14404)

[18] C.L. Mallows and N.J.A. Sloane, An upper bound for self-dual codes, Inform. Control 22 (1973), 188-200. MR0414223 (54:2326)

[19] G. Nebe, Finite subgroups of $\mathrm{GL}_{n}(\mathbf{Q})$ for $25 \leq n \leq 31$, Comm. Algebra 24 (1996), 2341-2397. MR:1390378(97e:20066)

[20] V. Pless, N.J.A. Sloane and H.N. Ward, Ternary codes of minimum weight 6 and the classification of length 20, IEEE Trans. Inform. Theory 26 (1980), 305-316. MR570014|(81b:94033)

[21] E. Rains and N.J.A. Sloane, "Self-dual codes," Handbook of Coding Theory, V.S. Pless and W.C. Huffman (Editors), Elsevier, Amsterdam 1998, pp. 177-294. MR.1667939

Department of Mathematical Sciences, Yamagata University, Yamagata 990-8560, JAPAN

Graduate School of Information Sciences, Tohoku University, Sendai 980-8579, Japan

Steklov Institute of Mathematics at St. Petersburg, St. Petersburg 191011, Russia 